\title{
Wheat streak mosaic virus Lacking Helper Component-Proteinase Is Competent to Produce Disease Synergism in Double Infections with Maize chlorotic mottle virus
}

\author{
Drake C. Stenger, Brock A. Young, Feng Qu, T. Jack Morris, and Roy French
}

\begin{abstract}
First, second, and fifth authors: U.S. Department of Agriculture-Agricultural Research Service (USDA-ARS), and Department of Plant Pathology, University of Nebraska, Lincoln 68583; and third and fourth authors: School of Biological Sciences, University of Nebraska, Lincoln, NE 68588.
\end{abstract}

Current address of first author: USDA-ARS, 9611 S. Riverbend Ave., Parlier, CA 93648.

Accepted for publication 25 April 2007.

\begin{abstract}
Stenger, D. C., Young, B. A., Qu, F., Morris, T. J., and French, R. 2007. Wheat streak mosaic virus lacking helper component-proteinase is competent to produce disease synergism in double infections with Maize chlorotic mottle virus. Phytopathology 97:1213-1221.

The tritimovirus Wheat streak mosaic virus (WSMV) and the machlomovirus Maize chlorotic mottle virus (MCMV) each cause systemic chlorosis in infected maize plants. Infection of maize with both viruses produces corn lethal necrosis disease (CLND). Here, we report that complete deletion of the WSMV helper component-proteinase (HC-Pro) coding region had no effect on induction of CLND symptoms following coinoculation of maize with WSMV and MCMV. We further demonstrated that elevation of virus titers in double infections, relative to single infections, also was independent of WSMV HC-Pro. Thus, unlike poty-

virus HC-Pro, WSMV HC-Pro was dispensable for disease synergism. Because disease synergism involving potyviruses requires HC-Promediated suppression of posttranscriptional gene silencing (PTGS), we hypothesized that WSMV HC-Pro may not be a suppressor of PTGS. Indeed, WSMV HC-Pro did not suppress PTGS of a green fluorescent protein (GFP) transgene in an Agrobacterium-mediated coinfiltration assay in which potyvirus HC-Pro acted as a strong suppressor. Furthermore, coinfiltration with potyvirus HC-Pro, but not WSMV HC-Pro, resulted in elevated levels of the GFP target mRNA under conditions which trigger PTGS. Collectively, these results revealed significant differences in HC-Pro function among divergent genera of the family Potyviridae and suggest that the tritimovirus WSMV utilizes a gene other than HC-Pro to suppress PTGS and mediate synergistic interactions with unrelated viruses.
\end{abstract}

Coinfection of plants by two unrelated viruses may result in disease synergism with symptoms more severe than the additive effects of each virus individually. Corn lethal necrosis disease (CLND) is a field example of synergism caused by double infection of maize with the machlomovirus Maize chlorotic mottle virus (MCMV) and one of several maize-infecting potyviruses $(13,25,41)$. Under laboratory conditions, the tritimovirus Wheat streak mosaic virus (WSMV) may be substituted for the potyvirus to experimentally generate CLND symptoms in double infections with MCMV (32). Separately, each virus induces systemic chlorosis but not necrosis. In contrast, infection of maize with both viruses causes extensive necrosis, stunting, and premature death of infected plants. Also, as with many synergistic interactions among unrelated plant viruses $(2,7,13,15,16,30,34,43$, 44,46 ), infection of maize with WSMV and MCMV may result in elevated virus titers compared with that observed in single infections (32).

Potyvirus helper component-proteinase (HC-Pro) mediates synergistic interactions among unrelated viruses in double infections $(14,28,31,33,44)$ by posttranscriptional gene silencing (PTGS) suppression $(1,6,8,20,22,45)$. Although WSMV HC-Pro shares cysteine proteinase functions in common with potyvirus HC-Pro (38) and is required for semipersistent transmission of WSMV by the wheat curl mite $(38,39)$, a role for tritimovirus

Corresponding author: D. C. Stenger; E-mail address: dstenger@fresno.ars.usda.gov

doi:10.1094/PHYTO-97-10-1213

This article is in the public domain and not copyrightable. It may be freely reprinted with customary crediting of the source. The American Phytopathological Society, 2007.
HC-Pro in disease synergism and suppression of PTGS has not been investigated. Recently, it was demonstrated (36) that WSMV tolerated complete deletion of the HC-Pro coding region (the $\mathrm{P} 1 / \mathrm{P} 3$ junction was cleaved by $\mathrm{P} 1$ proteinase) without alteration of pathogenicity or virulence in maize. This unexpected observation prompted us to hypothesize that tritimovirus HC-Pro function may differ from that of potyvirus HC-Pro with respect to synergism and PTGS suppression. In this study, we evaluated disease phenotype and relative virus titer in maize plants coinfected with MCMV and progeny virus derived from WSMV constructs encoding or lacking HC-Pro. We further evaluated WSMV HC-Pro function using a well established transient assay for suppression of PTGS (29).

\section{MATERIALS AND METHODS}

Virus isolates. The MCMV isolate used in these experiments was the same as that described by Goldberg and Brakke (13). MCMV was recovered from dried maize leaves (after nearly 20 years in storage at $4^{\circ} \mathrm{C}$ ) by sap inoculation of maize inbred line 'SDp2'. MCMV infection of SDp2 plants caused mild systemic mottling symptoms and was verified by reverse transcriptionpolymerase chain reaction (RT-PCR) using primers for the coat protein $(\mathrm{CP})$ gene (described below) based on the complete nucleotide sequence of MCMV (26; GenBank Accession no. NC_003627). The 673-bp RT-PCR product was cloned into pGEMT-easy (Promega, Madison, WI) and a consensus sequence (corresponding to MCMV nucleotides 3422 to 4094) based on four independent clones was compared with NC_003627. The consensus sequence was identical to that of the corresponding 
region of NC_003627, except for two synonymous C to U transitions at nucleotides 3704 and 3929.

The WSMV genotypes evaluated in this study were based on an infectious cDNA clone (10) of the WSMV-Sidney 81 isolate (37; GenBank accession no. NC_001886). Three WSMV constructs (Fig. 1A) modified from the original infectious clone (pACYCWSMV) were used as templates for in vitro transcription (10): pS81-SA (containing SalI and ApaI sites flanking the HC-Pro coding region), pS81-SAS12 (containing an additional SalI site immediately downstream of the ApaI site), and pS81 $\triangle \mathrm{HC} 12-5$ (completely lacking HC-Pro and derived from pS81-SAS12 by deletion of the SalI fragment). Transcripts derived from all three WSMV constructs were previously shown to be infectious to wheat and stable upon passage by sap transmission to both wheat and SDp2 maize $(35,36)$.

Establishment of single and double infections in maize inbred line SDp2. Symptomatic tissue $(\approx 2 \mathrm{~g})$ from leaves of SDp2 plants individually infected with MCMV or each WSMV genotype was ground in water $(40 \mathrm{ml})$ with a mortar and pestle. To establish single infections, $10 \mathrm{ml}$ of each inocula was diluted

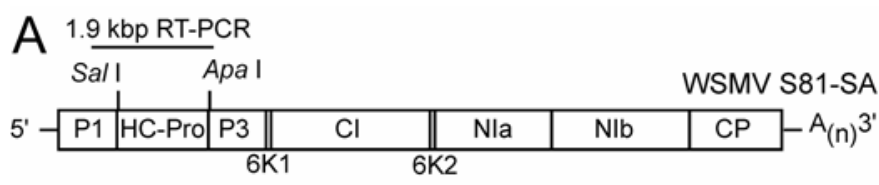



$0.8 \mathrm{kbp} \mathrm{RT}-\mathrm{PCR}$

\begin{tabular}{|c|c|c|c|c|c|c|}
\hline Sal I & & & & \multicolumn{3}{|c|}{ WSMV S81 $1 \mathrm{HC} 12-5$} \\
\hline P1 & P3 & $\mathrm{Cl}$ & $\mathrm{Nla}$ & $\mathrm{Nlb}$ & $\mathrm{CP}$ & $-A_{(n)^{3}}$ \\
\hline \multicolumn{7}{|c|}{$6 \mathrm{~K} 2$} \\
\hline & & & \multicolumn{2}{|c|}{ Replicase } & bp RT & PCR \\
\hline & & & p50 & p111 & $\mathrm{CP}$ & \multirow{2}{*}{ MCMV } \\
\hline & & & p32 & & p31 & \\
\hline
\end{tabular}

B pZP-GFP RB P35S TLL GFP pZP-TEV-HCPro RB P35S TL TEV HC-Pro T35S LB. pZP-WSMV-HCPro RB P35S TLL WSMV HC-Pro T35S LB.

pZP-Empty RB P35S T35S LB

Fig. 1. Virus genome maps and construct diagrams. A, Genome maps of Maize chlorotic mottle virus (MCMV) and three Wheat streak mosaic virus (WSMV) genotypes (S81-SA, S81-SAS12, and S81 $\Delta$ HC12-5) used in disease synergism assays. WSMV genotypes S81-SA and S81-SAS12 both encode helper component-proteinase (HC-Pro) and are identical except for a second SalI site engineered into S81-SAS12. WSMV genotype S81 $\Delta \mathrm{HC} 12-5$ lacks the HC-Pro coding region and was constructed by deletion of the SalI fragment of S81-SAS12. The location and size (in kilobase pairs) of reverse transcription-polymerase chain reaction products used to verify infection of plants are indicated above each genome map. B, Schematic representation of expression cassettes placed within the Agrobacterium tumefaciens binary shuttle vector pZP212 used in posttranscriptional gene silencing suppression assays. Abbreviations are as follows: right border (RB), tandem $35 \mathrm{~S}$ promoter (P35S), Tobacco etch virus RNA leader (TL), green fluorescent protein (GFP), Tobacco etch virus HC-Pro (TEV HC-Pro), Wheat streak mosaic virus HC-Pro (WSMV HC-Pro), 35S terminator (T35S), and left border (LB). with 1 volume of water and applied mechanically to five SDp2 seedlings (two- to three-leaf stage). To establish double infections of MCMV and each WSMV genotype, mixtures of paired inocula (10 ml each) were mechanically inoculated onto five SDp2 seedlings each. Celite was added to all inocula as an abrasive. Uninoculated SDp2 seedlings served as negative controls. Following mechanical inoculation, seedlings were rinsed with water and placed in a growth chamber $\left(16 \mathrm{~h}\right.$ light/day, $24^{\circ} \mathrm{C}$ during the day and $21^{\circ} \mathrm{C}$ at night). Verification of single and double infections was accomplished by enzyme-linked immunosorbent assay (ELISA) and RT-PCR (described below). The entire experiment was repeated except that plants were maintained under different lighting conditions ( $12 \mathrm{~h}$ light per day) in the second experiment. In both experiments, plants representative of each virus inoculation treatment held beyond 30 days postinoculation (dpi) were moved to a greenhouse (because of growth chamber limits on plant height) and monitored for symptom development through 50 dpi.

RT-PCR. Total nucleic acid samples were extracted (24) from symptomatic, systemically infected $\mathrm{SDp} 2$ leaf tissue $(\approx 0.5 \mathrm{~g})$. Complimentary DNA was synthesized by reverse transcription using total nucleic acid samples as templates and a mixture of reverse primers MCMVR and HCR as described (36). MCMVR annealed to MCMV RNA nucleotides 4094 to 4075, whereas HCR annealed to WSMV-Sidney 81 sequences immediately downstream of the HC-Pro coding region (nucleotides 2373 to 2345). PCR amplification of MCMV or WSMV sequences was accomplished in separate reactions using cDNA as a template with primers MCMVR and MCMVF (MCMV nucleotides 3422 to 3441) or primers HCR and P1V1 (WSMV-Sidney 81 nucleotides 421 to 445$)$. PCR conditions were as described previously (36) with the assays designed to be qualitative, not quantitative. Amplification of MCMV sequences was expected to generate an $\approx 0.7$-kbp product; amplification of WSMV sequences was expected to generate an $\approx 1.9$-kbp product for genotypes containing the HC-Pro coding region or an $\approx 0.8$-kbp product from the WSMV genotype lacking HC-Pro (Fig. 1A). For each plant evaluated for relative virus titer by ELISA (described below), infection status was independently verified by RT-PCR.

Estimation of relative virus titer in single and double infections. Commercially available double antibody sandwich ELISA kits (Agdia, Elkhart, IN) were used to determine relative titer of MCMV or WSMV (39). Systemically infected, symptomatic leaf tissue $(1 \mathrm{~g})$ was sampled at two time points: 17 and $30 \mathrm{dpi}$ in experiment 1 or 15 and $28 \mathrm{dpi}$ in experiment 2 . At each time point, tissue samples were collected from the second and third youngest leaves. This sampling strategy avoided necrotic areas that developed on older leaves of plants infected with both viruses.

Leaf samples were ground in $10 \mathrm{ml}$ of ELISA extraction buffer. A 2-ml aliquot of each ground sample was subjected to brief centrifugation $(30 \mathrm{~s}, 12,000 \times g)$, and the supernatant was serially diluted (twofold increments) in extraction buffer. Serially diluted samples (corresponding to 50, 25, 12.5, 6.25, 3.12, and $1.56 \mathrm{mg}$ of leaf tissue per $\mathrm{ml}$ ) were applied to microtiter plates previously coated with antibodies to MCMV or WSMV. For each virus treatment at each time point, a total of four samples (two from each of two plants) were tested with each antiserum. Different plants were sampled at each time point. ELISA absorbance values were recorded when a positive-reacting sample first achieved a value of $\approx 2.2$ optical density (OD) with MCMV antiserum or $\approx 1.7$ OD with WSMV antiserum. Corresponding mean absorbance values of uninfected samples were $\approx 0.10$ OD (MCMV antiserum) or $\approx 0.15$ OD (WSMV antiserum).

Within each time point, mean ELISA absorbance values for each sample dilution were plotted (after subtraction of mean absorbance values obtained with uninfected plant samples) on a log-log scale (e.g., mean ELISA absorbance value as a function of 
sample dilution). Relative titers of MCMV or WSMV were estimated for each virus treatment within a time point using the ELISA absorbance versus sample dilution plot of single infections with S81-SA or MCMV as standards. Thus, within each time point, virus titer of S81-SA or MCMV in single infections was assigned a value of $100 \%$ with titers of the remaining virus treatments for that time point estimated as a percentage relative to the appropriate standard. Each relative virus titer estimate was reported as the mean percentage of the appropriate standard based on mean ELISA absorbance values obtained for three or four serial dilutions that plotted within the range of the appropriate standard. Relative titers were only compared within a single time point as differences in absolute titer of samples used as standards at different time points were not assessed.

PTGS suppression assay. A transient in planta assay previously described by Qu et al. (29) was used to evaluate the potential of WSMV HC-Pro to suppress PTGS. Tobacco etch virus (TEV) HC-Pro served as a positive control in the PTGS suppression assay. The TEV HC-Pro coding region was amplified by PCR using pRTL2-0027 (provided by J. C. Carrington) as template; WSMV-Sidney 81 HC-Pro was amplified by PCR using pACYC-WSMV (10) as template. Both HC-Pro coding regions were amplified with virus-specific primers incorporating a start codon within a $B s p H I$ site ( $5^{\prime}$-proximal primers) and a stop codon followed by an XbaI site (WSMV) or KpnI site (TEV) (3'proximal primers). Primer sequences for TEV HC-Pro were $5^{\prime}$ TCATGAGCGACAAATCAATCTCTGAGGCATTCT-3' and ' 5'GGTACCTTATCCAACATTGTAAGTTTTCATTTC-3'; primer sequences for WSMV HC-Pro were 5'-TCATGAGCGGCGACCAAGCAGTGAACAAAG- $3^{\prime}$ and 5'-TCTAGATTAGCCAATTTTGTAATCTTTCATTG-3'. The resulting PCR products were cloned into pGEMT-easy and verified by sequencing of the cloned inserts. Each HC-Pro coding region was excised from pGEMTeasy with $B s p \mathrm{HI}$ and $X b a \mathrm{I}$ or $K p n \mathrm{I}$ and used to replace the NcoIXbaI or NcoI-KpnI fragment (containing the Turnip mosaic virus [TuMV] P1/HC-Pro coding region) of pRTL2-TuMV. The resulting constructs contained the TEV or WSMV HC-Pro coding region as part of an expression cassette (35S promoter/TEV leader/HC-Pro/35S terminator) that was excised by digestion of flanking PstI sites and positioned between the Agrobacterium tumefaciens T-DNA right and left borders (Fig. 1B) of the shuttle/binary plasmid pZP212 (provided by T. Clemente). The resulting pZP212 derivatives were electroporated into A. tumefaciens and selected by using the appropriate antibiotic regime. A. tumefaciens cultures were verified as harboring the appropriate pZP212 derivatives by PCR by using the HC-Pro primers described previously. Another pZP212 derivative containing only the $35 \mathrm{~S}$ promoter/TEV leader/35S terminator was constructed and placed into A. tumefaciens (29) and served as an "empty vector" negative control (Fig. 1B).

A. tumefaciens cultures were grown and prepared for infiltration as described by $\mathrm{Qu}$ et al. (29). Attached leaves of transgenic Nicotiana benthamiana plants (line $16 \mathrm{c}$ provided by D. C. Baulcombe) constitutively expressing green fluorescent protein (GFP) were infiltrated with A. tumefaciens cultures or mock infiltrated with cell resuspension buffer as a negative control. A. tumefaciens containing a pZP212 derivative expressing GFP (29; Fig. 1B) was infiltrated into line $16 \mathrm{c}$ leaves to initiate PTGS of the endogenous GFP transgene. To evaluate PTGS suppression activity, A. tumefaciens bearing the GFP expression cassette was coinfiltrated in combination with A. tumefaciens cultures bearing TEV HC-Pro or WSMV HC-Pro expression cassettes or the empty vector control. Infiltrated plants were maintained in a growth chamber $\left(12 \mathrm{~h} \mathrm{light} /\right.$ day, $24^{\circ} \mathrm{C}$ during the day and $21^{\circ} \mathrm{C}$ at night). Six days postinfiltration, GFP expression in the infiltrated leaves was assessed by fluorescence upon UV illumination. Images of UV-illuminated leaves were obtained with a digital camera by using both UV and yellow filters. For comparison, fluorescence of noninfiltrated leaves of nontransgenic $N$. benthamiana was photographed under the same conditions. GFP mRNA expression levels in infiltrated leaves were determined at 6 days postinfiltration by northern blotting of total RNA samples as described (29).

\section{RESULTS}

Symptom expression in single or double infections. The infection status of each inoculated SDp2 plant (five per treatment per replicate) was verified by qualitative RT-PCR assays during the course of both experiments. In all cases, RT-PCR indicated that plants inoculated with MCMV contained MCMV but not WSMV. Conversely, all plants inoculated with WSMV contained WSMV but not MCMV. All plants inoculated with mixtures of WSMV and MCMV contained both viruses. Because the PCR primers employed for WSMV detection flanked the HC-Pro coding region (Fig. 1A), the assay was able to distinguish infection by the HC-Pro deletion mutant $\mathrm{S} 81 \Delta \mathrm{HC} 12-5$ from the other two WSMV genotypes encoding HC-Pro (S81-SA and S81-SAS12) based on product size ( $0.8 \mathrm{kbp}$ versus $1.9 \mathrm{kbp}$, respectively). No RT-PCR products were observed for uninfected control plants. Representative RT-PCR assays of plants sampled from experiment 1 at 17 dpi are shown in Figure 2.

Plants singly infected with MCMV developed mild chlorotic mottling symptoms beginning $\approx 7$ to $10 \mathrm{dpi}$ with similar symptoms appearing on new leaves as they expanded through 50 dpi in both experiments. No substantial differences in the timing or severity of symptoms were observed among plants individually inoculated with each of the three WSMV genotypes through 50 dpi in both experiments. In single infections, each WSMV genotype induced typical systemic mosaic symptoms that first appeared $\approx 9$ to $13 \mathrm{dpi}$

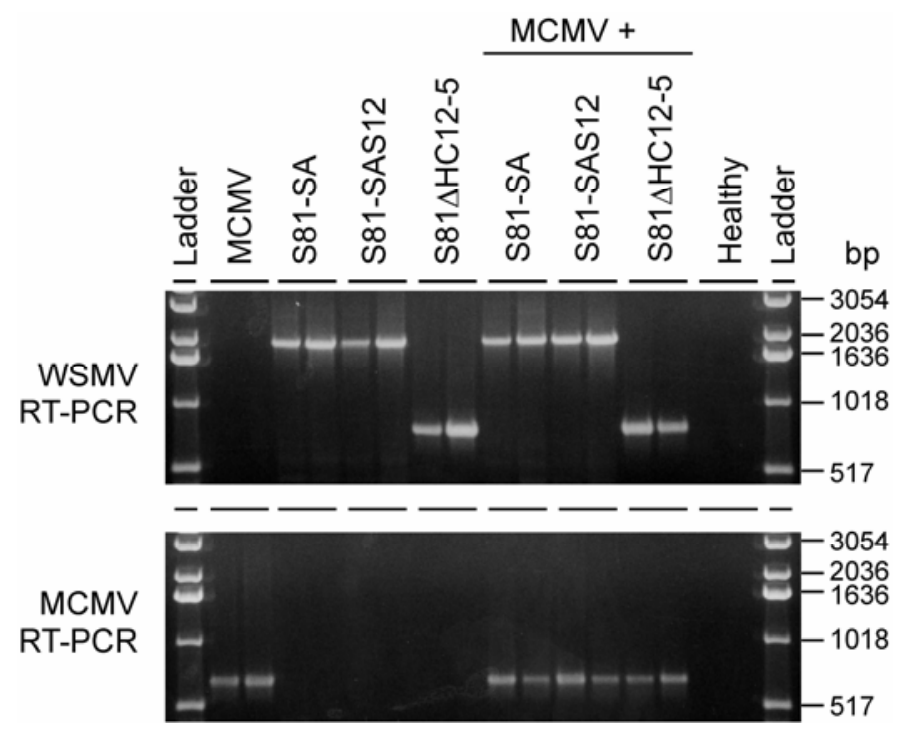

Fig. 2. Reverse transcription-polymerase chain reaction (RT-PCR) verification of single and double infections. Presented are images of 1\% agarose gels following electrophoresis of RT-PCR products amplified from SDp2 maize plants singly or doubly infected with Wheat streak mosaic virus (WSMV) and/or Maize chlorotic mottle virus (MCMV). Lane labels correspond to RTPCR amplification of samples (two each) extracted from uninfected plants (healthy) or plants infected with MCMV alone, with each of three WSMV genotypes (S81-SA, S81-SAS12, and S81 $\Delta \mathrm{HC12-5)}$ alone, or with MCMV and each of the three WSMV genotypes (MCMV+). Outer lanes (ladder) contain DNA standards with sizes indicated in kilobase pairs at right. RT-PCR was conducted using primers specific to WSMV (upper gel) or MCMV (lower gel). Amplification of MCMV generated a $0.7-\mathrm{kbp}$ product; amplification of WSMV generated a $1.9-\mathrm{kpb}$ product for genotypes encoding helper component-proteinase (HC-Pro) (S81-SA and S81-SAS12) or a 0.8-kbp product for S81 $\triangle \mathrm{HC} 12-5$ in which the HC-Pro coding region had been deleted. Note that the RT-PCR assay was designed to be qualitative and not quantitative. 

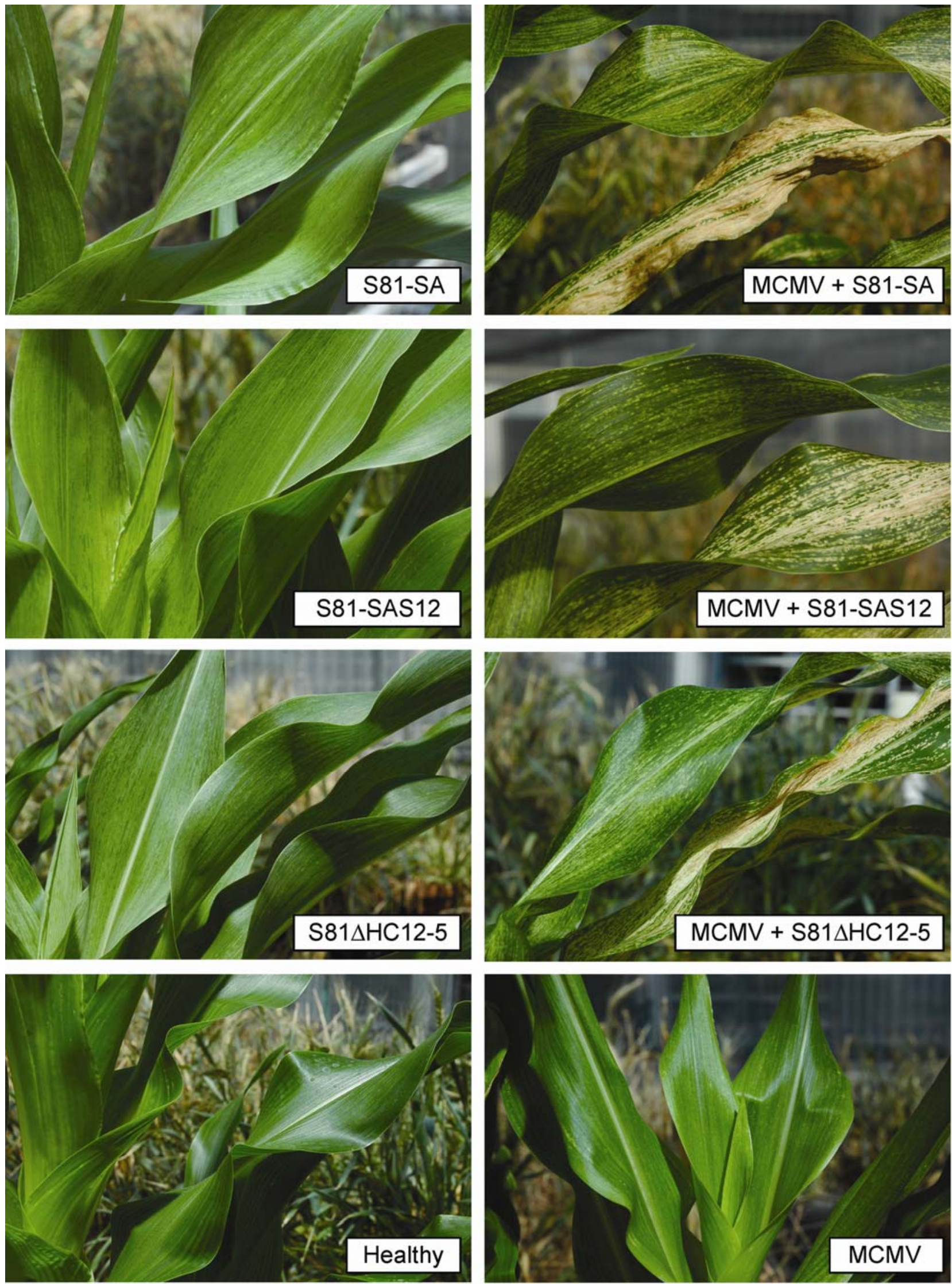

Fig. 3. Expression of corn lethal necrosis disease (CLND) symptoms 30 days postinoculation. Presented are images of SDp 2 maize plants singly or doubly infected with Wheat streak mosaic virus (WSMV) and/or Maize chlorotic mottle virus (MCMV). Plants singly infected with WSMV genotypes S81-SA, S81SAS12 (both encoding helper component-proteinase [HC-Pro]), or S81 $\triangle \mathrm{HC} 12-5$ (HC-Pro deleted) expressed systemic chlorotic streak mosaic symptoms; plants singly infected with MCMV developed mild systemic chlorotic mottling. Plants doubly infected with MCMV and each of the three WSMV genotypes exhibited severe CLND symptoms characterized by extensive regions of systemic necrosis on fully developed leaves. Note that development of CLND symptoms was independent of WSMV HC-Pro. Plant shown at lower left is an uninoculated (healthy) control. 
as isolated chlorotic streaks. At 15 to 17 dpi, plants singly infected with each WSMV genotype displayed more numerous, albeit still mostly separated, chlorotic streaks that by 28 to $30 \mathrm{dpi}$ had coalesced. At 50 dpi, plants singly infected with each of the WSMV genotypes continued to exhibit chlorotic mosaic symptoms on newly developed leaves. Throughout both experiments, plants singly infected with MCMV or each of the three WSMV genotypes did not develop systemic necrosis.

Enhanced severity and accelerated development of symptoms were observed for all doubly infected SDp2 plants, regardless of which WSMV genotype was paired with MCMV. Systemic chlorotic streaks first appeared on doubly infected plants $\approx 7$ to 9 dpi and were more numerous and of larger size compared with the initial systemic symptoms observed several days later for plants singly infected with WSMV. By 15 to 17 dpi, doubly infected plants were noticeably stunted, relative to singly infected plants, with patches of necrosis developing on older fully expanded leaves. By 28 to $30 \mathrm{dpi}$, doubly infected plants produced new growth with coalescent chlorotic streaks and extensive zones of necrotic tissue on older leaves. Figure 3 shows representative symptoms expressed by singly and doubly infected plants from experiment 1 at $30 \mathrm{dpi}$. By $50 \mathrm{dpi}$, singly infected plants reached the tassel growth stage with no evidence of systemic necrosis, whereas doubly infected plants failed to produce tassels, exhibited extensive systemic necrosis of leaves, and were moribund (Fig. 4).

Virus titers in single and double infections. Relative virus titers measured at two time points in each experiment are pre-

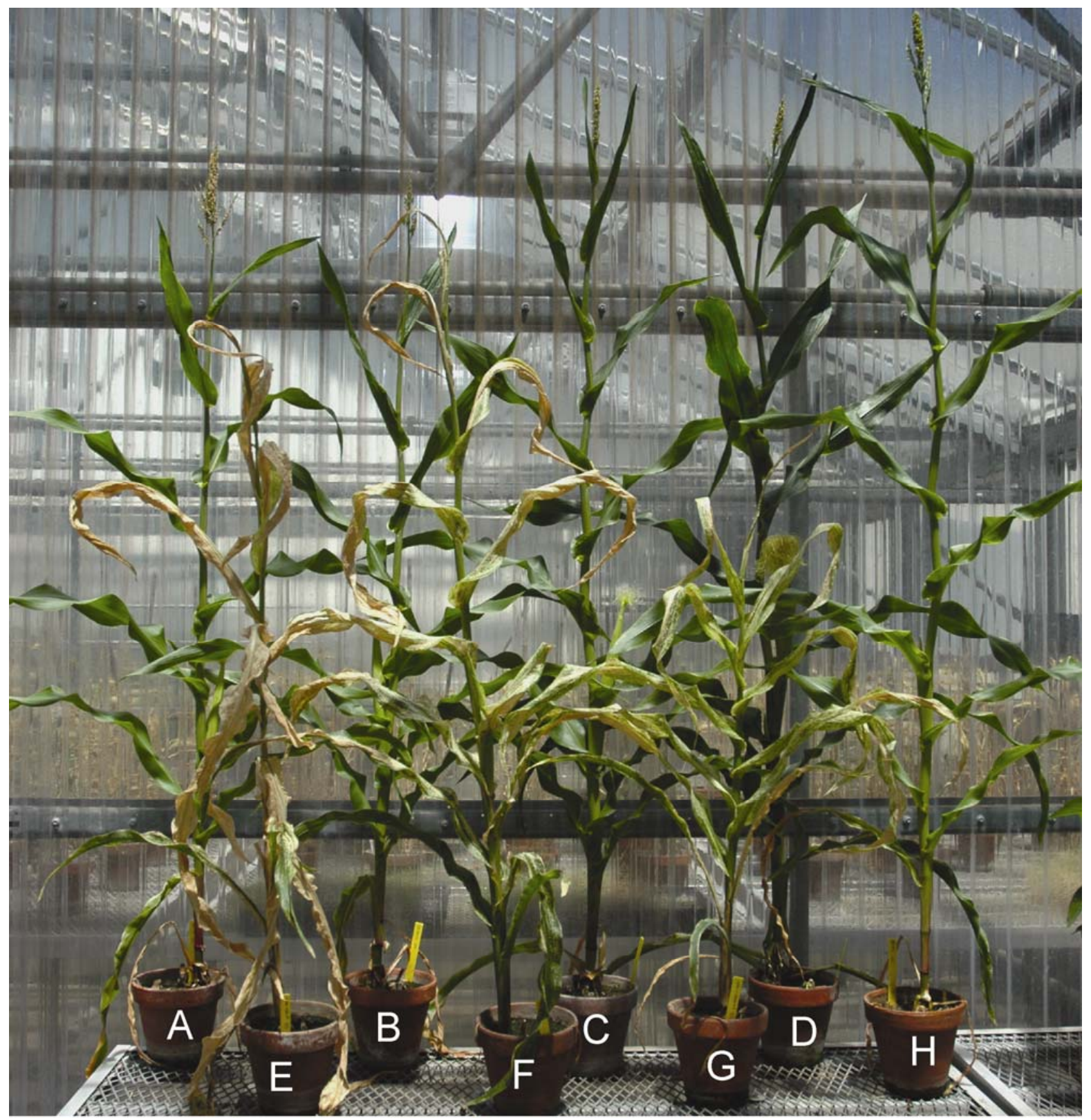

Fig. 4. Advanced symptoms of corn lethal necrosis disease (CLND) 50 days postinoculation. Presented are images of SDp2 maize plants singly or doubly infected with Wheat streak mosaic virus (WSMV) and/or Maize chlorotic mottle virus (MCMV). Plants A to C were singly infected with WSMV genotypes A, S81-SA or $\mathbf{B}$, S81-SAS12 encoding helper component-proteinase (HC-Pro), or $\mathbf{C}, \mathbf{S} 81 \Delta \mathrm{HC} 12-5$ lacking HC-Pro. Plant $\mathbf{D}$ is an uninfected SDp2 plant. Plants $\mathbf{E}$ to $\mathbf{G}$ were doubly infected with MCMV and WSMV genotypes E, S81-SA, F, S81-SAS12, or G, S81 $\triangle$ HC12-5 and exhibited CLND symptoms. Plant H was infected with MCMV only. Note that development of CLND symptoms was independent of WSMV HC-Pro. 
sented in Figure 5. In both experiments, there were minimal differences in titer among the three WSMV genotypes in single infections at 15 to $17 \mathrm{dpi}$. In contrast, titer of all three WSMV genotypes was increased approximately three- to sixfold in doubly infected plants at 15 to 17 dpi relative to S81-SA alone. However, at the second time point ( 28 to $30 \mathrm{dpi}$ ), relative titer of WSMV in doubly infected plants was not substantially greater than S81-SA alone and was, in most cases, less than that observed for S81-SA alone.

Coinfection with WSMV increased MCMV titer compared with single infections (Fig. 5). However, the mean increase in MCMV relative titer in doubly infected plants was less than twofold at the early time point (15 to $17 \mathrm{dpi}$ ) sampled in both experiments. At the later time point ( 28 to $30 \mathrm{dpi}$ ) sampled, mean relative titer of

Exp 1
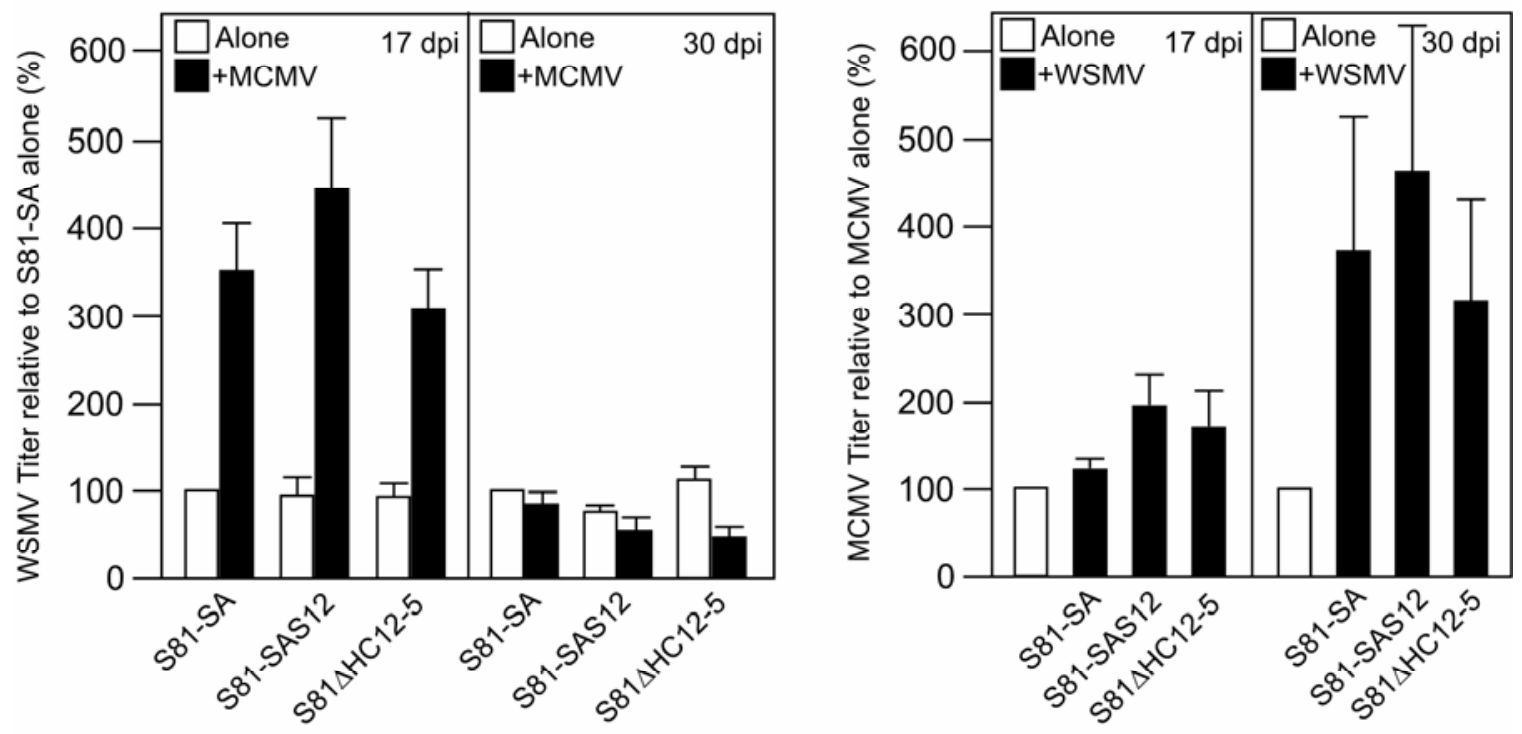

$\operatorname{Exp} 2$
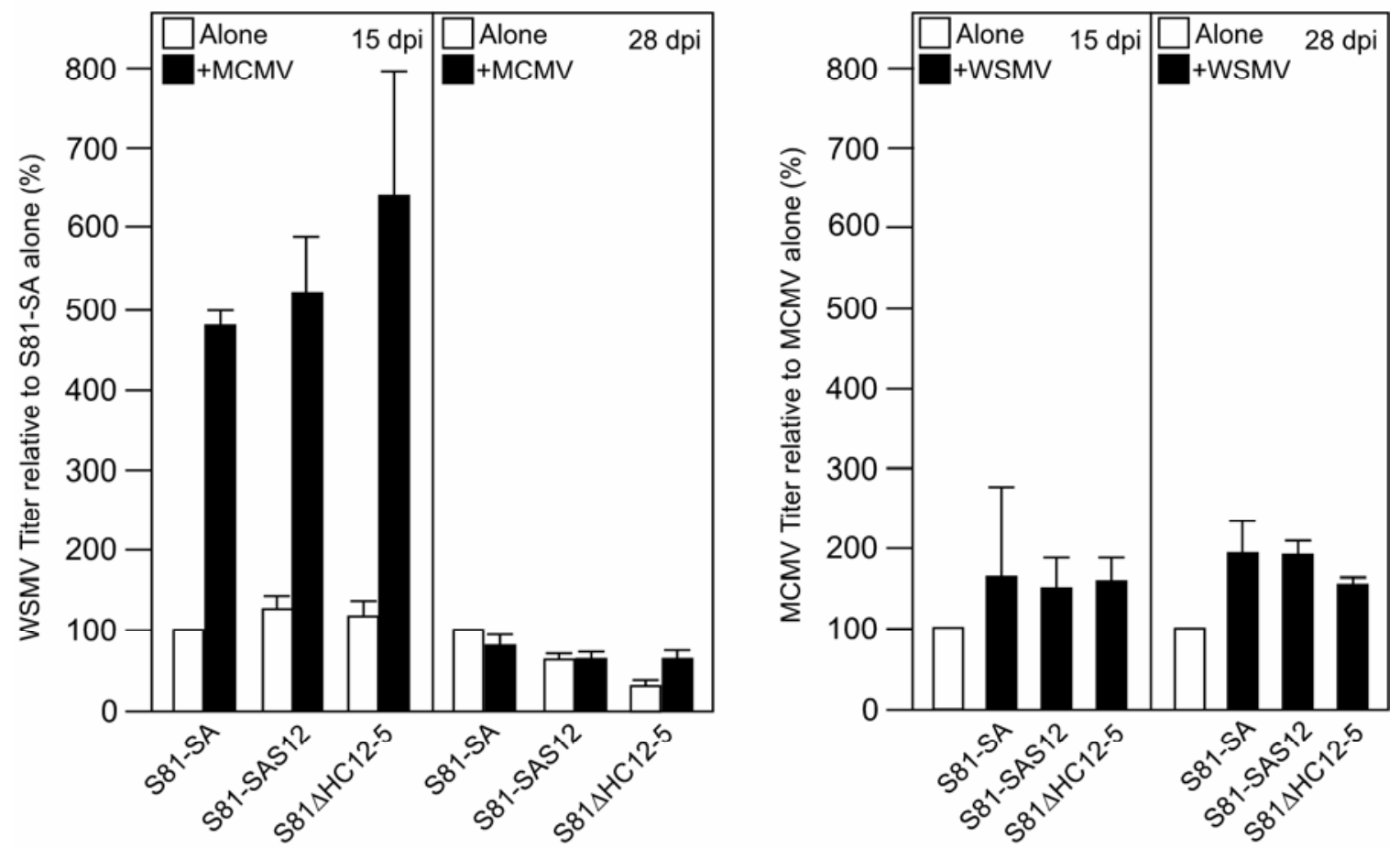

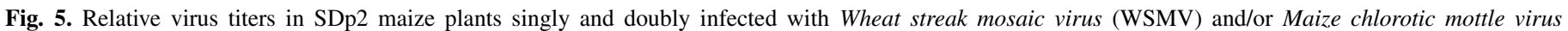



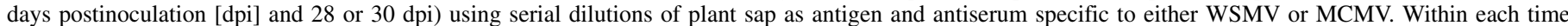

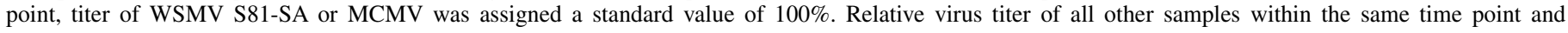

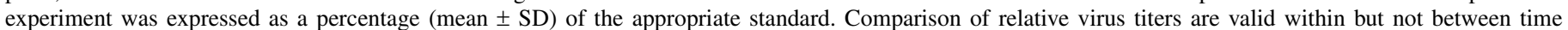

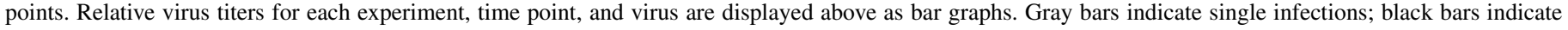

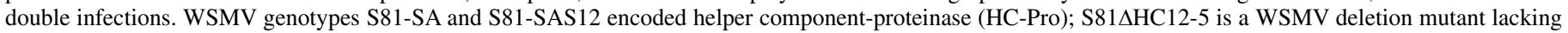

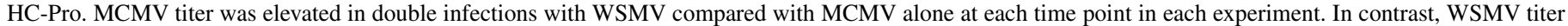

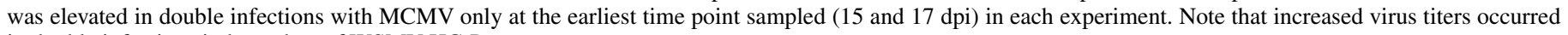
in double infections independent of WSMV HC-Pro. 
MCMV in doubly infected plants was three- to fourfold greater than for MCMV alone in one experiment, but less than twofold greater in the second experiment. Despite this variation, elevation of MCMV titer in double infections with WSMV was independent of HC-Pro.

WSMV HC-Pro lacks PTGS suppression activity. PTGS of the GFP transgene was induced upon infiltration of $N$. benthamiana line $16 \mathrm{c}$ leaves with A. tumefaciens bearing the GFP expression cassette but not by infiltration of cell resuspension buffer (Fig. 6). Coinfiltration of GFP transgenic plants with the A. tumefaciens culture containing the GFP expression cassette and a second A. tumefaciens culture expressing TEV HC-Pro resulted in high levels of GFP expression 6 days after infiltration, confirming TEV HC-Pro as a strong suppressor of PTGS. In contrast, an A. tumefaciens culture expressing WSMV HC-Pro did not exhibit PTGS suppression activity upon coinfiltration with the A. tumefaciens culture containing the GFP expression cassette (Fig. 6). Similarly, no PTGS suppression activity was detected when the GFP expressing A. tumefaciens culture was coinfiltrated with $A$. tumefaciens containing the empty vector control. In northern blots (Fig. 7), GFP mRNA levels were increased (as expected) by coinfiltration with $A$. tumefaciens expressing TEV HC-Pro but not by coinfiltration with A. tumefaciens expressing WSMV HC-Pro.

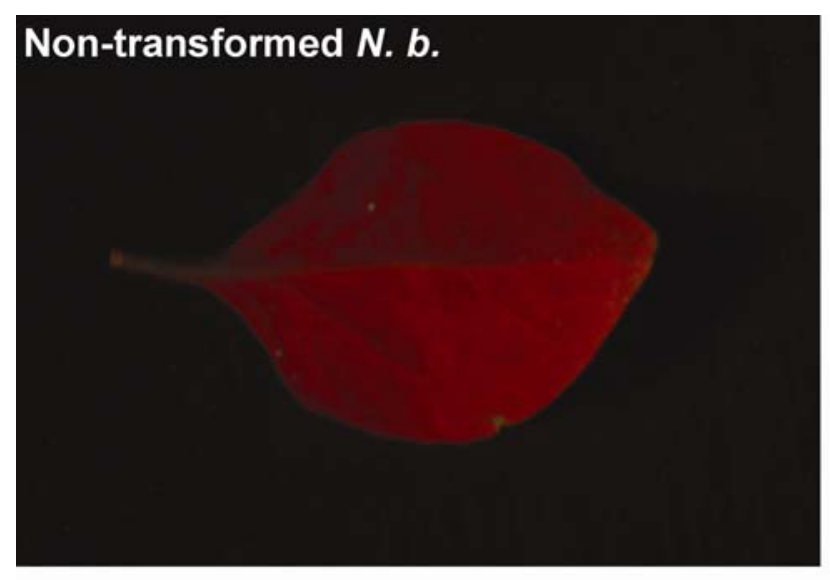

16 N. ..

\section{Buffer only}

16c N. b.

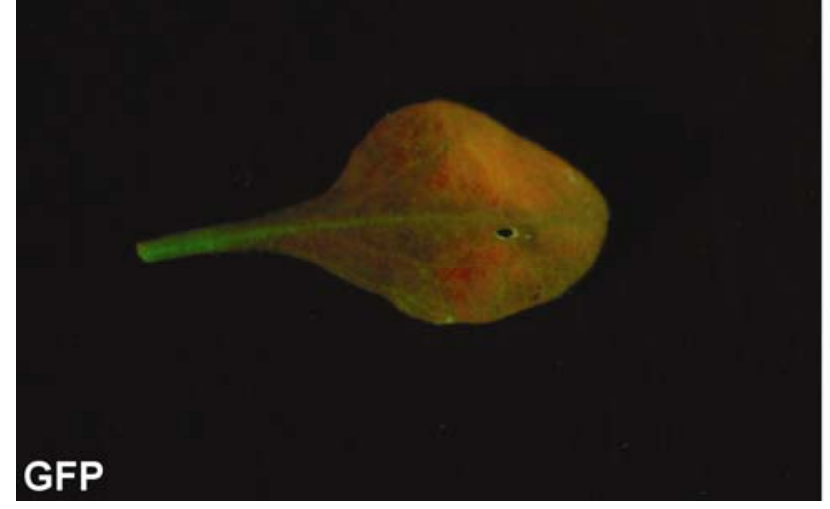

\section{$16 c$ N. b.}

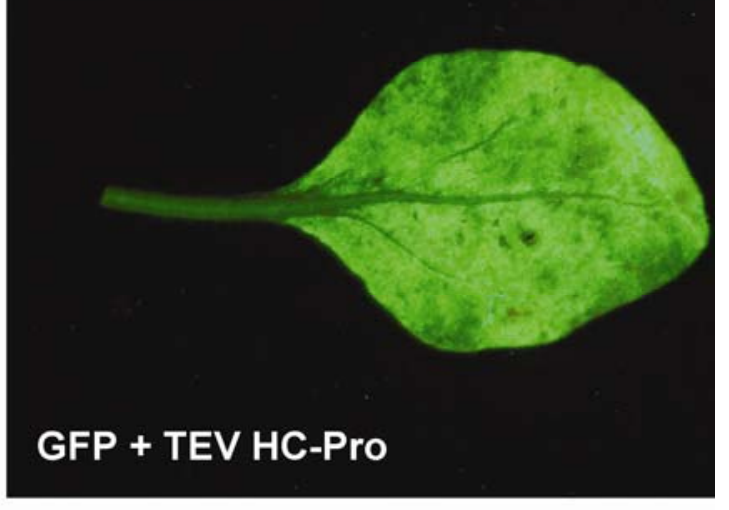

16 N. $b$.
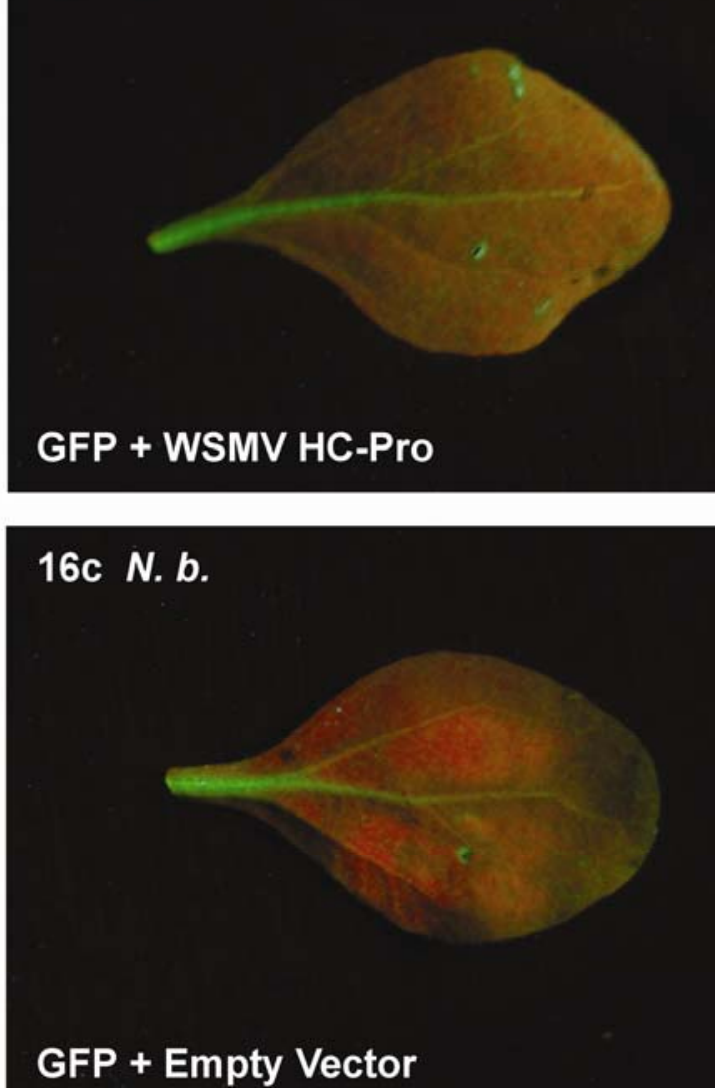

Fig. 6. Evaluation of Wheat streak mosaic virus (WSMV) helper component-proteinase (HC-Pro) in a posttranscriptional gene silencing suppression assay. Presented are images (photographed under UV illumination) of Nicotiana benthamiana leaves lacking green fluorescent protein (GFP) (nontransformed $N$. b.) or containing a constitutively expressed GFP transgene $(16 \mathrm{c} N$. b.) 6 days postinfiltration. Leaves were infiltrated with cell resuspension buffer (buffer only), or infiltrated with Agrobacterium tumefaciens cultures harboring binary Ti plasmids bearing an expression cassette for GFP alone or coinfiltrated with expression cassettes for GFP and Tobacco etch virus (TEV) HC-Pro, WSMV HC-Pro, or the Ti binary plasmid lacking an inserted gene (empty vector). 


\section{DISCUSSION}

WSMV HC-Pro is dispensable for disease synergism. We successfully reproduced CLND in double infections of maize with WSMV and MCMV, whether or not the particular WSMV genotype encoded HC-Pro. The extensive necrosis, stunting, and premature death of doubly infected plants clearly satisfied the classic definition of disease synergism. We further confirmed the observation of Scheets (32) that WSMV virus titer was enhanced in doubly infected plants. However, in our experiments, WSMV titer was higher in doubly infected plants at 2 weeks but not 1 month after inoculation. We also found that titer of MCMV was greater in plants infected with both viruses compared with MCMV alone and, although the increase was of smaller magnitude than observed previously (32), the response was independent of WSMV HC-Pro. As Scheets (32) reproduced CLND symptoms in a different maize line (N28Ht) using a different isolate of MCMV (MCMV-Kansas) paired with WSMV-Sidney 81 and evaluated disease synergism under different environmental conditions, it is perhaps not surprising that some variation among the two studies was encountered. Nonetheless, our results clearly indicated that WSMV HC-Pro was not required for disease synergism.

Does WSMV encode a suppressor of PTGS? WSMV HC-Pro was unable to function as a suppressor of PTGS under conditions in which potyvirus HC-Pro was a strong suppressor of PTGS. This result can be interpreted in two ways: either WSMV HC-Pro does not suppress PTGS or the assay employed was not adequate to detect suppressor activity of WSMV HC-Pro. Although not all viral encoded suppressors of PTGS function in the Agrobacterium-mediated coinfiltration assay used here (23), many do, including an ever-increasing array of orthologous PTGS suppressors from unrelated viruses of both plants $(9,17,19,23,29)$ and animals (21). Given that WSMV lacking HC-Pro remained competent to induce disease synergism with MCMV and since disease synergism in other systems is dependent on PTGS suppression $(6,8,14,20,45)$, the simplest interpretation is that WSMV HC-Pro is not a suppressor of PTGS.

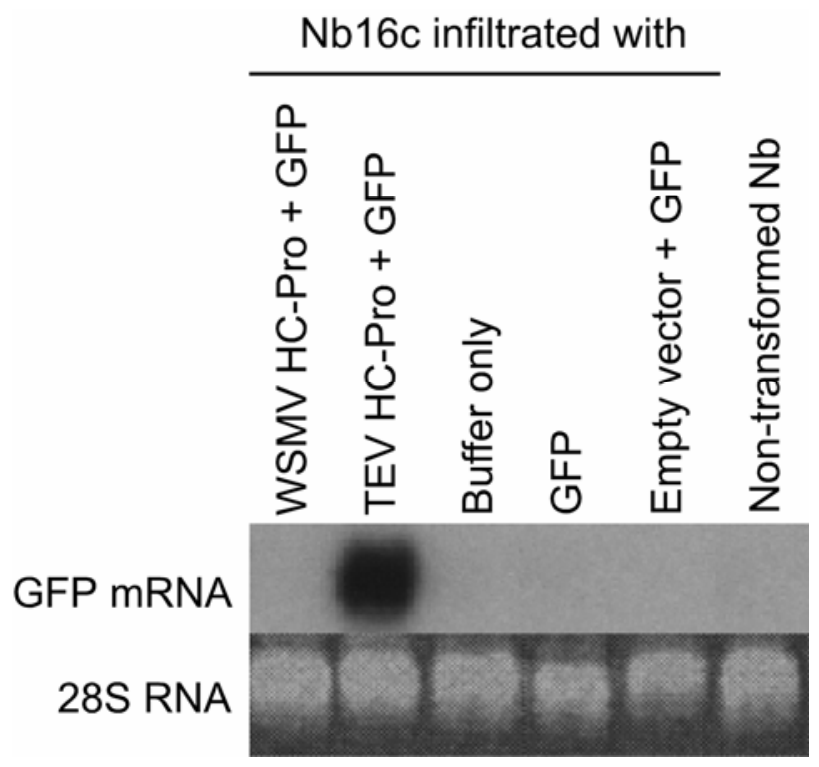

Fig. 7. Effect of Wheat streak mosaic virus (WSMV) helper component-proteinase (HC-Pro) on green fluorescent protein (GFP) mRNA levels in a posttranscriptional gene silencing (PTGS) suppression assay. Presented is a northern blot probed with ${ }^{32} \mathrm{P}$-labeled GFP DNA (upper panel) of total RNA samples $(5 \mu \mathrm{g})$ extracted from Nicotiana benthamiana $(\mathrm{Nb}) 16 \mathrm{c}$ leaves 6 days postinfiltration. Note that Tobacco etch virus (TEV) HC-Pro, but not WSMV HC-Pro, suppressed PTGS as evidenced by high levels of GFP mRNA. Ethidium bromide-stained 28S rRNA (lower panel) in total RNA samples served as loading controls.
The data reported here revealed significant differences in $\mathrm{HC}$ Pro function among genera and lead to an obvious and testable hypothesis: Are PTGS suppression and disease synergism functions of a WSMV gene other than HC-Pro? Alternatively, but viewed by us as less probable, WSMV may not need a suppressor of PTGS for systemic infection of plants and induction of disease synergism. Given that Cucumber vein yellowing virus (a whiteflytransmitted species of the genus Ipomovirus) lacks an HC-Pro homologue and instead utilizes an imperfect repeat of P1 (designated as P1b) to accomplish PTGS suppression (42), the former hypothesis seems more likely. To address this, we are currently examining other WSMV genes for PTGS suppression and pathogenicity enhancement activity.

Tritimovirus and potyvirus HC-Pro are divergent in both sequence and function. The family Potyviridae contains a diverse collection of virus species assigned to six genera (4). Among these, at least four genera encode proteins designated as HC-Pro homologues: Potyvirus, Tritimovirus, Ipomovirus (e.g., some isolates of Sweet potato mild mottle virus), and Rymovirus. However, HC-Pro encoded by different genera share limited sequence identity, and alignment of HC-Pro amino acid sequences outside of the carboxy-terminal cysteine proteinase domain are mostly ambiguous and of low confidence (12).

Aside from recent experiments with WSMV (this report, 35,36,38-40), functional analyses of HC-Pro have been restricted to potyviruses. Nonetheless, there seems little doubt that HC-Pro of all four genera retain cysteine proteinase functions needed for polyprotein processing, as conserved motifs of the cysteine proteinase active site and cleavage site are readily identifiable in sequence comparisons. Furthermore, cysteine proteinase activity has been demonstrated directly for WSMV HC-Pro (38). Although vector transmission determinants map to the amino-terminal region of HC-Pro encoded by both potyviruses $(3,5,11,18,27)$ and tritimoviruses $(38,39)$, viruses of these two genera have distinctly different vector taxa and modes of transmission, making it unlikely that the exact same mechanism is employed. Whether other functions of HC-Pro demonstrated for potyviruses are conserved among genera remains an open question. Thus, systematic and comprehensive studies of HC-Pro encoded by genera of the family Potyviridae are needed to assess evolutionary relationships in a functional context.

\section{ACKNOWLEDGMENTS}

We thank D. C Baulcombe for sharing $N$. benthamiana line 16c; J. C. Carrington and T. Clemente for providing various plasmids; and K. Stenberg for technical assistance. Mention of proprietary or brand names are necessary to report factually on available data; however, the USDA neither guarantees nor warrants the standard of the product, and the use of the name by USDA implies no approval to the exclusion of others that also may be suitable. This article is in the public domain and not copyrightable. It may be freely reprinted with customary crediting of the source.

\section{LITERATURE CITED}

1. Anandalakshmi, R., Pruss, G. J., Ge, X., Marathe, R., Mallory, A. C., Smith, T. H., and Vance, V. B. 1998. A viral suppressor of gene silencing in plants. Proc. Natl. Acad. Sci. USA 95:13079-13084.

2. Anjos, J. R., Jarflors, U., and Ghabrial, S. A. 1992. Soybean mosaic potyvirus enhances the titer of two comoviruses in dually infected soybean plants. Phytopathology 82:1022-1027.

3. Atreya, P. L., Lopez-Moya, J. J., Chu, M. H., Atreya, C. D., and Pirone, T. P. 1995. Mutational analysis of the coat protein N-terminal amino-acids involved in potyvirus transmission by aphids. J. Gen. Virol. 76:265-270.

4. Berger, P. H., Adams, M. J., Barnett, O. W., Brunt, A. A., Hammond, J., Hill, J. H., Jordan, R. L., Kashiwazaki, S., Rybicki, E., Spence, N., Stenger, D. C., Ohki, S. T., Uyeda, I., van Zaayen, A., Valkonen, J., and Vetten, H. J. 2005. Family Potyviridae. Pages 819-841 in: The International Committee on the Taxonomy of Viruses, 8th Report. C. M. Fauquet. C. M. Fauquet, M. A. Mayo, J. Maniloff, U. Desselberger, and L. 
A. Ball, eds. Elsevier/Academic Press, London.

5. Blanc, S., Ammar, E. D., Garcia-Lampasona, S., Dolja, V. V., Llave, C., Baker, J., and Pirone, T. P. 1998. Mutations in the potyvirus helper component protein: Effects on interactions with virions and aphid stylets. J. Gen. Virol. 79: 3119-3122.

6. Brigneti, G., Voinnet, O., Li, W.-X., Ji, L.-H., Ding, S.-W., and Baulcombe, D. C. 1998. Viral pathogenicity determinants are suppressors of transgene silencing in Nicotiana benthamiana. EMBO J. 17:6739-6746.

7. Calvert, L. A., and Ghabrial, S. A. 1983. Enhancement by soybean mosaic virus of bean pod mottle virus titer in doubly infected soybean. Phytopathology 73:992-997.

8. Carrington, J. C., Kasschau, K. D., and Johansen, L. K. 2001. Activation and suppression of RNA silencing by plant viruses. Virology 281:1-5.

9. Chiba, M., Reed, J. C., Prokhnevsky, A. I., Chapman, E. J., Mawassi, M., Koonin, E. V., Carrington, J. C., and Dolja, V. V. 2006. Diverse suppressors of RNA silencing enhance agroinfection by a viral replicon. Virology 346:7-14.

10. Choi, I.-R., French, R., Hein, G. L., and Stenger, D. C. 1999. Fully biologically active in vitro transcripts of the eriophyid mite-transmitted wheat streak mosaic tritimovirus. Phytopathology 89:1182-1185.

11. Dolja, V. V., Herndon, K. L., Pirone, T. P., and Carrington, J. C. 1993. Spontaneous mutagenesis of a plant potyvirus genome after insertion of a foreign gene. J. Virol. 67:5968-5975.

12. French, R., and Stenger, D. C. 2005. Complete nucleotide sequences of Agropyron mosaic virus and Hordeum mosaic virus support reciprocal monophyly of the genera Potyvirus and Rymovirus in the family Potyviridae. Arch. Virol. 150:299-312.

13. Goldberg, K. A., and Brakke, M. K. 1987. Concentration of maize chlorotic mottle virus increased in mixed infections with maize dwarf mosaic virus, strain B. Phytopathology 77:162-177.

14. González-Jara, P., Atencio, F. A., Martínez-García, B., Barajas, D., Tenllado, F., and Díaz-Ruíz, J. R. 2005. A single amino acid mutation in the Plum pox virus helper component-proteinase gene abolishes both synergistic and RNA silencing suppression activities. Phytopathology 95:894-901.

15. Goodman, R. M., and Ross, A. F. 1974. Enhancement of potato virus X synthesis in doubly infected tobacco occurs in doubly infected cells. Virology 58:16-24.

16. Goodman, R. M., and Ross, A. F. 1974. Enhancement by potato virus Y of potato virus $\mathrm{X}$ synthesis of doubly infected tobacco depends on timing of invasion by the viruses. Virology 58:263-271.

17. Guo, H. S., and Ding, S. W. 2002. A viral protein inhibits the long range signaling activity of the gene silencing signal. EMBO J. 21:398-407.

18. Huet, H., Gal-on, A., Meir, E., Lecoq, H., and Raccah, B. 1994. Mutations in the helper component protease gene of zucchini yellow mosaic virus affect its ability to mediate aphid transmissibility. J. Gen. Virol. 75:14071414.

19. Johansen, L. K., and Carrington, J. C. 2001. Silencing on the spot. Induction and suppression of RNA silencing in the Agrobacteriummediated transient expression system. Plant Physiol. 126:930-938.

20. Kasschau, K. D., and Carrington, J. C. 1998. A counterdefensive strategy of plant viruses: Suppression of posttranscriptional gene silencing. Cell 95:461-470.

21. Li, H., Li, W. X., and Ding, S. W. 2003. Induction and suppression of RNA silencing by an animal virus. Science 296:1319-1321.

22. Llave, C., Kasschau, K. D., and Carrington, J. C. 2000. Virus-encoded suppressor of posttranscriptional gene silencing targets a maintenance step in the silencing pathway. Proc. Natl. Acad. Sci. USA 97:1340113406.

23. Lu, R., Folimonov, A., Shintaku, M., Li, W.-X., Falk, B. W., Dawson, W. O., and Ding, S. W. 2005. Three distinct suppressors of RNA silencing encoded by a $20-\mathrm{kb}$ viral RNA genome. Proc. Natl. Acad. Sci. USA 101:15742-15747.

24. McNeil, J. E., French, R., Hein, G. L., Baenziger, P. S., and Eskridge, K. M. 1996. Characterization of genetic variability among natural populations of wheat streak mosaic virus. Phytopathology 86:1222-1227.

25. Niblett, C. L., and Clafin, L. E. 1978. Corn lethal necrosis - A new virus disease of corn in Kansas. Plant Dis. Rep. 62:15-19.
26. Nutter, R. C., Scheets, K., Panganiban, L. C., and Lommel, S. A. 1989. The complete nucleotide sequence of the maize chlorotic mottle virus genome. Nucleic Acids Res. 17:3163-3177.

27. Peng, Y. H., Kadoury, D., Gal-On, A., Wang, Y., and Raccah, B. 1998. Mutations in the HC-Pro gene of zucchini yellow mosaic potyvirus: Effects on aphid transmission and binding to purified virions. J. Gen. Virol. 79:897-904.

28. Pruss, G., Ge, X., Shi, X. M., Carrington, J. C., and Vance, V. B. 1997. Plant viral synergism: The potyviral genome encodes a broad-range pathogenicity enhancer that transactivates replication of heterologous viruses. Plant Cell 9:859-868.

29. Qu, F., Ren, T., and Morris, T. J. 2003. The coat protein of turnip crinkle virus suppresses posttranscriptional gene silencing at an early initiation step. J. Virol. 77:511-522.

30. Rochow, W. F., and Ross, A. F. 1955. Virus multiplication in plants doubly infected by potato viruses $\mathrm{X}$ and Y. Virology 1:10-27.

31. Sáenz, P., Quiot, L., Quiot, J.-B., Candresse, T., and García, J. A. 2001. Pathogenicity determinants in the complex virus population of a Plum pox virus isolate. Mol. Plant-Microbe Interact. 14:278-287.

32. Scheets, K. 1998. Maize chlorotic mottle machlomovirus and wheat streak mosaic rymovirus concentrations increase in the synergistic disease corn lethal necrosis. Virology 242:28-38.

33. Shi, X. M., Miller, H., Verchot, J., Carrington, J. C., and Vance, V. B. 1997. Mutations in the region encoding the central domain of helper component-proteinase (HC-Pro) eliminate potato virus $\mathrm{X} /$ potyviral synergism. Virology 231:35-42.

34. Sonoda, S., Koiwa, H., Kanda, K., Kato, H., Shimono, M., and Nishiguchi, M. 2000. The helper component-proteinase of Sweet potato feathery mottle virus facilitates systemic spread of Potato virus $X$ in Ipomoea nil. Phytopathology 90:944-950.

35. Stenger, D. C., and French, R. 2004. Functional replacement of Wheat streak mosaic virus HC-Pro with the corresponding cistron from a diverse array of viruses in the family Potyviridae. Virology 323:257-267.

36. Stenger, D. C., French, R., and Gildow, F. E. 2005. Complete deletion of Wheat streak mosaic virus HC-Pro: A null mutant is viable for systemic infection. J. Virol. 79:12077-12080.

37. Stenger, D. C., Hall, J. S., Choi, I.-R., and French, R. 1998. Phylogenetic relationships within the family Potyviridae: Wheat streak mosaic virus and brome streak mosaic virus are not members of the genus Rymovirus. Phytopathology 88:782-787.

38. Stenger, D. C., Hein, G. L., and French, R. 2006. Nested deletion analysis of Wheat streak mosaic virus HC-Pro: Mapping of domains affecting polyprotein processing and eriophyid mite transmission. Virology 350:465-474.

39. Stenger, D. C., Hein, G. L., Gildow, F. E., Horken, K. M., and French, R. 2005. Plant virus HC-Pro is a determinant of eriophyid mite transmission. J. Virol. 79:9054-9061.

40. Stenger, D. C., Young, B. A., and French, R. 2006. Random mutagenesis of wheat streak mosaic virus HC-Pro: Non-infectious interfering mutations in a gene dispensable for systemic infection of plants. J. Gen. Virol. $87: 2741-2747$

41. Uyemoto, J. K. 1983. Biology and control of maize chlorotic mottle virus. Plant Dis. 67:7-10.

42. Valli, A., Martin-Hernadez, A. M., Lopez-Moya, J. J., and Garcia, J. A. 2006. RNA silencing suppression by a second copy of the P1 serine protease of Cucumber vein yellowing ipomovirus, a member of the family Potyviridae that lacks the cysteine protease HC-Pro. J. Virol. 80:1005510063.

43. Vance, V. B. 1991. Replication of potato virus X is altered in coinfections with potato virus Y. Virology 182:486-494.

44. Vance, V. B., Berger, P. H., Carrington, J. C., Hunt, A. G., and Shi, X. M. 1995. 5'-Proximal potyviral sequences mediate potato virus $\mathrm{X} /$ potyviral synergistic disease in transgenic tobacco. Virology 206:583-590.

45. Vance, V. B., and Vaucheret, H. 2001. RNA silencing in plants - Defense and counterdefense. Science 292:2277-2280.

46. Wintermantel, W. M. 2005. Co-infection of Beet mosaic virus with beet yellowing viruses leads to increased symptom expression on sugar beet. Plant Dis. 89:325-331 